\title{
GENERAL DORROH EXTENSIONS
}

\author{
I. ALHRIBAT, P. JARA, AND A. I. MÁRQUEZ*
}

\begin{abstract}
In a recent paper G. A. Cannon and K. M. Neuerburg point out that if $A=\mathbb{Z}$ and $B$ is an arbitrary ring with unity, then $\mathbb{Z} \star B$, the Dorroh extension of $B$, is isomorphic to the direct product $\mathbb{Z} \times B$. Thus, the ideal structure of $\mathbb{Z} \star B$ can be completely described. The aim of this note is to point out that this result may be extended to any pair $(A, B)$ in which $B$ is an $A$-algebra with unity, and to study the construction of extensions of algebras without zero divisors and their behavior with respect to algebra maps.
\end{abstract}

\section{Dorroh Extensions}

All rings $A$ have unity. We call a rng or a pseudo-ring any triad $(B,+, \times)$ in which $(B,+)$ is an abelian group, and $\times$ is associative and distributive with respect to + , i.e. a ring not necessarily with unity. By an $A$-algebra we mean a pseudo-ring $B$ with left and right $A$-module structures satisfying the following three properties:

$$
a_{1}\left(b a_{2}\right)=\left(a_{1} b\right) a_{2}, \quad a\left(b_{1} b_{2}\right)=\left(a b_{1}\right) b_{2}, \quad \text { and } \quad\left(b_{1} b_{2}\right) a=b_{1}\left(b_{2} a\right),
$$

for any $a, a_{1}, a_{2} \in A$ and $b, b_{1}, b_{2} \in B$. If in addition $B$ has a unity, say $e$, one may prove that $a e=e a$ for any $a \in A$; this means that the map $f: A \rightarrow B$, defined as $f(a)=a e$, is a ring map from $A$ to $B$. We define an $A$-algebra map from an algebra $B$ to an algebra $B^{\prime}$ as a two-sided module map $f: B \rightarrow B^{\prime}$ satisfying $f\left(b_{1} b_{2}\right)=f\left(b_{1}\right) f\left(b_{2}\right)$ for any $b_{1}, b_{2} \in B$. In the same way we may define left and right or two-sided ideals, subalgebras, and other elements and constructions generalizing those studied in ring theory. In this note we shall consider non-zero algebras, but it may be that the zero algebra $\{0\}$ appears inside some constructions.

Example 1.1. Let $A$ be a ring. The following are examples of $A$-algebras: (1) The ring $A$ is a unitary A-algebra.

(2) The set $\left\{\left(\begin{array}{ll}a & b \\ 0 & 0\end{array}\right) \mid a, b \in A\right\}$ is an A-algebra with a left sided unity.

*Partially supported by MTM2007-66666, FQM-266, and MTM2010-20940. 


\section{GENERAL DORROH EXTENSIONS}

(3) If $A$ is commutative and $a \in A$, then $a A$ is an $A$-algebra. For instance $X \mathbb{Z}[X]$ and $2 \mathbb{Z}$ are $\mathbb{Z}$-algebras.

(4) If $\left\{A_{\lambda} \mid \lambda \in \Lambda\right\}$ is a family of $A$-algebras, then $\oplus_{\lambda} A_{\lambda}$ and $\prod_{\lambda} A_{\lambda}$ are $A$-algebras. In this case $\oplus_{\lambda} A_{\lambda}$ is a subalgebra and a two-sided ideal of $\prod_{\lambda} A_{\lambda}$.

A classical problem in ring theory is how to embed an $A$-algebra $B$ in an $A$-algebra with unity, say $A \star B$, in such a way that this embedding is minimal in the following sense: for any unitary $A$-algebra $C$, any algebra map $f: B \rightarrow C$ may be uniquely factorized through $A \star B$. To construct $A \star B$ we proceed as follows.

For any $A$-algebra $B$, in the cartesian product underlying set $A \times B$ we define an addition by

$$
\left(a_{1}, b_{1}\right)+\left(a_{2}, b_{2}\right)=\left(a_{1}+a_{2}, b_{1}+b_{2}\right), \text { for any } a_{1}, a_{2} \in A \text { and } b_{1}, b_{2} \in B,
$$

and a multiplication by

$$
\begin{aligned}
& \left(a_{1}, b_{1}\right)\left(a_{2}, b_{2}\right)=\left(a_{1} a_{2}, a_{1} b_{2}+b_{1} a_{2}+b_{1} b_{2}\right), \\
& \text { for any } a_{1}, a_{2} \in A \text { and } b_{1}, b_{2} \in B .
\end{aligned}
$$

With these operations $A \times B$ is an $A$-algebra with unity $(1,0)$. We denote this $A$-algebra by $A \star B$ and call it the Dorroh extension ${ }^{1}$ of $B$ by $A$. If we identify $B$ with $\{(0, b) \mid b \in B\}$ and $A$ with $\{(a, 0) \mid a \in A\}$, then $B$ is an ideal and $A$ a unitary subalgebra of $A \star B$.

Theorem 1.2. For any $A$-algebra $B$, any unitary $A$-algebra $C$, and any algebra map $f: B \rightarrow C$, there exists a unique unitary algebra map $f^{\prime}$ : $A \star B \rightarrow C$ such that $f_{\mid B}^{\prime}=f$.

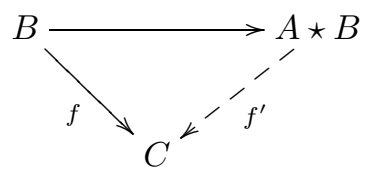

Note that any ring $B$ is a $\mathbb{Z}$-algebra. Hence, as we remarked before, the first construction involving Dorroh extensions deals with pairs $(\mathbb{Z}, B)$, see [3]. In this particular case, in [1] and [2] the ideal structure of $\mathbb{Z} \star \mathbb{Z}$ is studied. In the former paper it is pointed out that $\mathbb{Z} \star B$ is isomorphic to the direct product $\mathbb{Z} \times B$, for any ring $B$ with identity.

We are interested in the general case, in which $A$ may be different from $\mathbb{Z}$, because it allows us to use some useful tools of general ring theory.

Proposition 1.3. For any ring $A$ and any unitary $A$-algebra $B$ the Dorroh extension $A \star B$ is isomorphic to the direct product $A \times B$.

\footnotetext{
${ }^{1}$ This construction was first performed by J. L. Dorroh in [3] for $A=\mathbb{Z}$.
}

MISSOURI J. OF MATH. SCI., FALL 2015 


\section{ALHRIBAT, P. JARA, AND A. I. MÁRQUEZ}

Proof. Let $B$ be an algebra with unity, say $e \in B$, then $(0, e)$ is a central idempotent element in $A \star B$. Indeed, for any $(a, b) \in A \star B$ we have

$$
(a, b)(0, e)=(0, a e+b e)=(0, e a+e b)=(0, e)(a, b) .
$$

If we identify $e$ with $(0, e)$, then $B=e(A \star B)$. On the other hand $1=$ $(1,0)=(1,-e)+(0, e)$; this means that any element $(a, b) \in A \star B$ may be written as $(a, b)=(a,-a e)+(0, a e+b)$, and we have the decomposition $A \star B=(1-e)(A \star B)+e(A \star B)$ which produces the algebra isomorphism $A \star B=(1-e)(A \star B) \times e(A \star B)$.

As we have noted before, $e(A \star B)=B$. On the other hand, (1 $e)(A \star B)=\{(a,-a e) \mid a \in A\}$ is isomorphic to $A$ via the ring map $(a,-a e) \mapsto a$. As a consequence $A \star B \cong A \times B$ via the isomorphism $\varphi:(a, b) \mapsto(a, a e+b)$.

Note that even if $B$ is a unitary $A$-algebra, the Dorroh extension of $B$ does not coincide with $B$.

Some remarks on the ideal structure of $A \star B$. Note that if $B$ has a unity, ideals in $A \star B$ have a simple description. Indeed, for any ideal $\mathfrak{c} \subseteq A \star B$ we have the decomposition $\mathfrak{c}=(1-e) \mathfrak{c} \oplus e \mathfrak{c}$. Thus we proceed as follows:

$$
A \star B=(1-e)(A \star B) \oplus e(A \star B) \longrightarrow A \times B .
$$

$$
\mathfrak{c} \longrightarrow(1-e) \mathfrak{c} \oplus e \mathfrak{c} \longrightarrow \mathfrak{a} \times \mathfrak{b} .
$$

In this case we denote $\mathfrak{a}$ the ideal $p r_{A}(\mathfrak{c})$, where $p r_{A}: A \star B \rightarrow A$ is the projection. Therefore we have $(1-e) \mathfrak{c}=\{(a,-a e) \mid a \in \mathfrak{a}\} \rightarrow p r_{A}(\mathfrak{c})=\mathfrak{a}$. The ideal ec may also be completely determined; in particular $\mathfrak{b}=e \mathfrak{c} \subseteq B$, and it may be described as $\mathfrak{b}=\left\{p r_{B}(c)+p r_{A}(c) e \mid c \in \mathfrak{c}\right\}$. Therefore $\mathfrak{c}$ is determined by its images by the projections $p r_{A}$ and $p r_{B}$, and we have $\mathfrak{c}=\left\{\left(p r_{A}(c),-p r_{A}(c) e\right) \mid c \in \mathfrak{c}\right\} \oplus\left\{\left(0, p r_{B}(c)+p r_{A}(c) e\right) \mid c \in \mathfrak{c}\right\}$.

Are ideals in $A \star B$ of the type $\mathfrak{a} \star \mathfrak{b}$ (defined in the obvious way) for ideals $\mathfrak{a} \subseteq A$ and $\mathfrak{b} \subseteq B$ ? To answer this question first we note that for ideals $\mathfrak{a} \subseteq A$ and $\mathfrak{b} \subseteq B$ the following statements are equivalent: (a) $\mathfrak{a} \star \mathfrak{b} \subseteq A \star B$ is an ideal and (b) $B \mathfrak{a}, \mathfrak{a} B \subseteq \mathfrak{b}$. In this case we have

$$
\mathfrak{a} \star \mathfrak{b}=(1-e)(\mathfrak{a} \star \mathfrak{b}) \oplus e(\mathfrak{a} \star \mathfrak{b}) \rightarrow \mathfrak{a} \times(\mathfrak{a} e+\mathfrak{b})=\mathfrak{a} \times \mathfrak{b} .
$$

In general, given an ideal $\mathfrak{c} \subseteq A \star B$ we have $\mathfrak{c}=\mathfrak{a} \star \mathfrak{b}$, for some ideals $\mathfrak{a} \subseteq A$ and $\mathfrak{b} \subseteq B$, if and only if $\operatorname{pr}_{A}(\mathfrak{c}) e \subseteq p r_{B}(\mathfrak{c})$.

Prime ideals in $A \star B$ are completely determined as they are in $A \times B$. Prime ideals in $A \times B$ are either $A \times \mathfrak{b}$, with $\mathfrak{b} \subseteq B$ being prime, or $\mathfrak{a} \times B$, with $\mathfrak{a} \subseteq A$ being prime. On one hand we have the ideal $\{(a,-a e) \mid a \in A\} \oplus \mathfrak{b}$ 


\section{GENERAL DORROH EXTENSIONS}

(note that $A e \nsubseteq \mathfrak{b}$ as $\mathfrak{b} \subseteq B$ is a proper ideal), and on the other we have the ideal $\{(a,-a e) \mid a \in \mathfrak{a}\} \oplus B=\mathfrak{a} \star B$.

\section{Szendrei Extensions}

Let us consider the following examples.

Example 2.1. (1) If $A=\mathbb{Z}$ and $B=X \mathbb{Z}[X]$, the Dorroh extension is isomorphic to $\mathbb{Z}[X]$.

(2) If $A=\mathbb{Z}$ and $B=2 \mathbb{Z}$, the Dorroh extension has an underlying structure of an abelian group isomorphic to $\mathbb{Z} \times \mathbb{Z}$, hence it is not isomorphic to $\mathbb{Z}$.

(3) In general we have proved that if $B$ is a ring with identity, the Dorroh extension $Z \star B$ is isomorphic to $Z \times B$. Consider the case of $\mathbb{Z} \star \mathbb{Z}[X]$, which is isomorphic to $\mathbb{Z} \times \mathbb{Z}[X]$.

We note that in some cases the "smallest" unitary algebra containing $B$ is different of $A \star B$. The reason is that in the universal property in Theorem (1.2) characterizing Dorroh extensions, the map $f$ was an arbitrary (not necessarily unitary) algebra map. One may pose the question: is it possible to modify the Dorroh construction to some specific classes of algebras to construct "smallest" unitary algebra extensions?

One of these modifications is known as the Szendrei Extension, see [4]. We present here a modification of it. Let $B$ be an $A$-algebra such that $b B=0$ implies $b=0$ for every $b \in B$; we say $B$ is a proper algebra; prime and semiprime $A$-algebras are proper. Define $\mathfrak{b}=\{x \in A \star B \mid x B=0\}$.

Lemma 2.2. With the above notation, if $B$ is a proper algebra, $\mathfrak{b} \subseteq A \star B$ is a two-sided ideal and $\mathfrak{b} \cap B=0$.

In this case the composition map $B \rightarrow A \star B \rightarrow(A \star B) / \mathfrak{b}$ is injective, and we may identify $B$ with its image in $(A \star B) / \mathfrak{b}$. We call $(A \star B) / \mathfrak{b}$ the Szendrei Extension of $B$.

Note that if $B$ is a unitary $A$-algebra, it is a proper algebra and $(A \star B) / \mathfrak{b}$ and $B$ are isomorphic as unitary algebras. In particular, in the examples mentioned in (2.1) we have:

Example 2.3. (1) $(\mathbb{Z} \star X \mathbb{Z}[X]) / \mathfrak{b} \cong \mathbb{Z}[X]$.

(2) $(\mathbb{Z} \star 2 \mathbb{Z}) / \mathfrak{b} \cong \mathbb{Z}$.

(3) $(\mathbb{Z} \star B) / \mathfrak{b} \cong B$.

Does this mean that Szendrei Extensions are more useful than Dorroh Extensions? The answer is no, as the construction of Szendrei Extensions may dampen some of the functorial properties of Dorroh Extensions. In practice this is exactly what happens. For that reason it is not of interest from a categorial point of view. Nevertheless we have the following useful

MISSOURI J. OF MATH. SCI., FALL 2015 


\section{ALHRIBAT, P. JARA, AND A. I. MÁRQUEZ}

property of Szendrei Extensions, which indicates that it may be of interest from an arithmetical point of view.

Theorem 2.4. Let $A$ be a ring, $B$ a proper $A$-algebra, and $C$ a unitary $A$-algebra without zero divisors. For any non-zero algebra map $f: B \rightarrow C$ there is a unique unitary algebra map $f^{\prime \prime}:(A \star B) / \mathfrak{b} \rightarrow C$ such that $f_{\mid B}^{\prime \prime}=f$.

Proof. By Theorem (1.2), there is a unique unitary algebra map $f^{\prime}: A \star B \rightarrow$ $C$ such that $f_{\mid B}^{\prime}=f$. To prove this theorem we only need to prove that $f^{\prime}(\mathfrak{b})=0$. Indeed, if $x \in \mathfrak{b}$, then $x B=0$, hence, $0=f^{\prime}(x B)=f^{\prime}(x) f(B)$. Since $f(B) \neq 0$, then $f^{\prime}(x)=0$. Therefore, there is a unique unitary algebra map $f^{\prime \prime}:(A \star B) / \mathfrak{b} \rightarrow C$ such that $f_{\mid B}^{\prime \prime}=f$.

The following example shows that the absence of zero divisor in $C$ is necessary in the above theorem.

Example 2.5. Let us consider $A=\mathbb{Z}$, and $B=2 \mathbb{Z}$. In $A \star B=\mathbb{Z} \star 2 \mathbb{Z}$ the annihilator of $2 \mathbb{Z}$ is $\mathfrak{b}=\{(-2 x, 2 x) \mid x \in \mathbb{Z}\}$. If we take $C=\mathbb{Z}_{8}$ and $f: 2 \mathbb{Z} \rightarrow \mathbb{Z}_{8}$, defined by $f(2)=\overline{4}$; the only unitary algebra map extending $f$ to $\mathbb{Z} \star 2 \mathbb{Z}$ is $f^{\prime}: \mathbb{Z} \star 2 \mathbb{Z} \rightarrow \mathbb{Z}_{8}$, defined by $f^{\prime}(a, 2 b)=\overline{a+4 b}$ for any $a, b \in \mathbb{Z}$. As a consequence $f^{\prime}$ can't be extended to $(\mathbb{Z} \star 2 \mathbb{Z}) / \mathfrak{b}$, as $f^{\prime}(\mathfrak{b}) \neq 0$; indeed we have: $f^{\prime}(-2,2)=\overline{-2+4}=\overline{2} \neq 0$.

As an application of this Szendrei Extension, we have the original result due to Szendrei, see [4]. In it the author studied when the extension of a (commutative) domain is also a unitary domain (one of the main problems on Dorroh Extensions is the appearance of zero divisors), and the corresponding universal property for this construction.

Corollary 2.6. Let $A$ be a commutative ring, $B$ a prime commutative $A-$ algebra, and $C$ a prime unitary commutative $A$-algebra. Then $(A \star B) / \mathfrak{b}$ is a prime unitary commutative $A$-algebra, containing $B$ as an ideal, and for any non-zero algebra map $f: B \rightarrow C$ there is a unique unitary algebra map $f^{\prime \prime}:(A \star B) / \mathfrak{b} \rightarrow C$ such that $f_{\mid B}^{\prime \prime}=f$.

And we have an extension in the following sense.

Corollary 2.7. Let $A$ be a commutative ring, $B$ a semiprime commutative $A$-algebra, and $C$ a semiprime unitary commutative $A$-algebra. Then $(A \star$ $B) / \mathfrak{b}$ is a semiprime unitary commutative $A$-algebra, containing $B$ as an ideal, and for any non-zero algebra map $f: B \rightarrow C$ there is a unique unitary algebra map $f^{\prime \prime}:(A \star B) / \mathfrak{b} \rightarrow C$ such that $f_{\mid B}^{\prime \prime}=f$.

Proof. Following with the notation in the proof of Theorem (2.4), let $\mathcal{P}=$ $\{\mathfrak{p} \mid \mathfrak{p} \subseteq C\}$ the set of all prime ideals of $C$, then we consider the following 


\section{GENERAL DORROH EXTENSIONS}

commutative diagram.

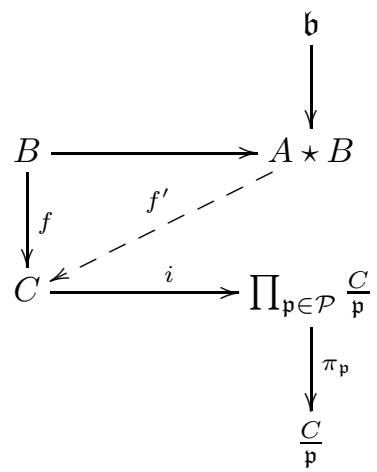

Where $\pi_{\mathfrak{p}}: \prod_{\mathfrak{p} \in \mathcal{P}} \frac{C}{\mathfrak{p}} \rightarrow \frac{C}{\mathfrak{p}}$ is the canonical projection on the factor, for any $\mathfrak{p} \in \mathcal{P}$. Since $\pi_{\mathfrak{p}} \circ i \circ f^{\prime}(\mathfrak{b})=0$, for every prime ideal $\mathfrak{p} \in \mathcal{P}$, then $i \circ f^{\prime}(\mathfrak{p})=0$, i.e. $f^{\prime}(\mathfrak{b})=0$, and this provides the existence of a unique $A$-algebra map $f^{\prime \prime}:(A \star B) / \mathfrak{b} \rightarrow C$ such that $f_{\mid B}^{\prime \prime}=f$.

From now on, let us consider a commutative ring $A$ and commutative $A$-algebras. Let $f: B_{1} \rightarrow B_{2}$ be a map between proper commutative $A-$ algebras. It is well-known that $f$ induces a map of unitary $A$-algebras: $A \star f: A \star B_{1} \rightarrow A \star B_{2}$, defined in the obvious way. Is the same result for Szendrei Extensions true? The answer is no, as the following example shows.

Example 2.8. Let us consider $A=\mathbb{Z}, B_{1}=2 \mathbb{Z}, B_{2}=\mathbb{Z}_{8}[X]$, and $f: 2 \mathbb{Z} \rightarrow$ $\mathbb{Z}_{8}[X]$ defined as $f(2)=\overline{4}$. The Szendrei Extension of $2 \mathbb{Z}$ is $(\mathbb{Z} \star 2 \mathbb{Z}) / \mathfrak{b}_{1}$, where $\mathfrak{b}_{1}=\{(-2 n, 2 n) \in \mathbb{Z} \star 2 \mathbb{Z} \mid n \in \mathbb{Z}\}$, and the Szendrei Extension of $\mathbb{Z}_{8}[X]$ is $\left(\mathbb{Z} \star \mathbb{Z}_{8}[X]\right) / \mathfrak{b}_{2} \cong \mathbb{Z}_{8}[X]$, where $\mathfrak{b}_{2}=\left\{(a, \bar{b}) \in \mathbb{Z} \star \mathbb{Z}_{8}[X] \mid \bar{a}+\bar{b}=0\right\}$. The map $\mathbb{Z} \star f$ is defined as $(\mathbb{Z} \star f)(a, 2 n)=(a, \overline{4 n})$. Since $(-2,2) \in \mathfrak{b}_{1}$, and $(\mathbb{Z} \star f)(-2,2)=(-2, \overline{4}) \notin \mathfrak{b}_{2}$ as $\overline{-2}+\overline{4}=\overline{-2+4}=\overline{2} \neq 0$. As a consequence, $f$ does not induce a unitary A-algebra map.

We have a positive answer in several particular cases. Thus, $f$ induces a unitary $A$-algebra map whenever either $f: B_{1} \rightarrow B_{2}$ is surjective or $f$ is a unitary $A$-algebra map.

On one hand, if $f$ is a surjective $A$-algebra map and $(a, b) \in \mathfrak{b}_{1}$ then $(a, b) B_{1}=0$, hence, $(a, f(b)) B_{2}=(a, f(b)) f\left(B_{1}\right)=A \star f\left((a, b) B_{1}\right)=0$, and $f$ induces a surjective $A$-algebra map $f^{\prime}:\left(A \star B_{1}\right) / \mathfrak{b}_{1} \rightarrow\left(A \star B_{2}\right) / \mathfrak{b}_{2}$. On the other hand, in the unitary case, $f^{\prime}$ coincides with $f$.

If $f: B_{1} \rightarrow B_{2}$, an $A$-algebra map between proper commutative $A_{-}$ algebras with kernel $\mathfrak{a}$, induces a map $f^{\prime}:\left(A \star B_{1}\right) / \mathfrak{b}_{1} \rightarrow\left(A \star B_{2}\right) / \mathfrak{b}_{2}$, it is straightforward to prove the following result.

MISSOURI J. OF MATH. SCI., FALL 2015 


\section{ALHRIBAT, P. JARA, AND A. I. MÁRQUEZ}

Proposition 2.9. With the above notation the kernel of $f^{\prime}$ is $\frac{\left(\mathfrak{a}: B_{1}\right)}{\mathfrak{b}_{1}}$, where $\left(\mathfrak{a}: B_{1}\right)=\left\{x \in A \star B_{1} \mid x B_{1} \subseteq \mathfrak{a}\right\}$. In particular, the kernel of $f^{\prime}$ contains $\frac{\mathfrak{a}+\mathfrak{b}_{1}}{\mathfrak{b}_{1}}$.

Then in general, we have the following corollary.

Corollary 2.10. Let $f: B_{1} \rightarrow B_{2}$ be an A-algebra map between proper commutative $A$-algebras with kernel $\mathfrak{a}$ such that $B_{2}$ is prime. Then $A \star$ $B_{2} / \mathfrak{b}_{2}$ is a prime ring, there is a map $f^{\prime}:\left(A \star B_{1}\right) / \mathfrak{b}_{1} \rightarrow\left(A \star B_{2}\right) / \mathfrak{b}_{2}$, and $\frac{\left(\mathfrak{a}: B_{1}\right)}{\mathfrak{b}_{1}} \subseteq \frac{A \star B_{1}}{\mathfrak{b}_{1}}$ is a prime ideal.

\section{REFERENCES}

[1] G. A. Cannon and K. M. Neuerburg, Ideals in Dorroh extensions of rings, Missouri J. Math. Sci., 20.3 (2008), 165-168.

[2] T. de Alwis, The ideal structure of $\mathbb{Z} \star \mathbb{Z}$, Missouri J. Math. Sci., 6.2 (1994), 116-123.

[3] J. L. Dorroh, Concerning adjunctions to algebras, Transactions of the Amer. Math. Soc., 26 (1932), 85-88.

[4] J. Szendrei, On the extension of rings without divisors of zero, Acta. Univ. Szegred, 13 (1950), 231-234.

MSC2010: 16D25, 16S70.

Key words: ring, algebra, Dorroh extension, Szendrei extension.

Department of Algebra, University of Granada, 18071 - Granada, Spain

E-mail address: xxxx@ugr.es

Department of Algebra, University of Granada, 18071 - Granada, Spain

E-mail address: pjara@ugr.es

Department of Algebra, University of Granada, 18071 - Granada, Spain

E-mail address: xxxx@ugr.es 